\title{
Die Entgrenzung industrieller Beziehungen: Vielfalt und Öffnung als neues Potential für Interessenvertretung?
}

\author{
Von Manfred Deiß und Klaus Schmierl
}

\begin{abstract}
Zusammenfassung: Entgrenzungs- und Erosionsprozesse bringen zugleich neue Grenzziehungen und neuartige institutionelle Strukturierungen mit sich. Im vorliegenden Beitrag wird widersprüchlichen Effekten der Entgrenzung und Institutionalisierung im deutschen System industrieller Beziehungen nachgegangen. Hierzu werden Erscheinungsformen der Auflösung und der Emergenz von Grenzziehungen in den Grundlagen und Strukturen inner- und überbetrieblicher Interessenvertretung der Arbeitnehmer einander gegenübergestellt, um Anhaltspunkte zur Beantwortung folgender Fragen zu gewinnen: Inwieweit wird das System industrieller Beziehungen durch Entgrenzungsprozesse lediglich zwischen traditionellen und stabilen, wenn auch tendenziell an Bedeutung verlierenden Formen einerseits und neuartigen, institutionell unterschiedlichen Mustern der Interessenvertretung andererseits weiter aufgefächert? Treibt diese Aufweichung der tradierten Strukturen die industriellen Beziehungen zu einer intransparenten Vielfalt mit erheblichen Wirksamkeitsverlusten oder bilden sich neuartige, stabilisierende Elemente heraus, die sich als (Re-)Vitalisierung der Interessenvertretung von Arbeitnehmern auswirken können?
\end{abstract}

\section{Einleitung}

Entgrenzung hat Konjunktur. Überall werden Prozesse der Entgrenzung beobachtet, in allen Gesellschaftsbereichen erodieren bisher selbstverständliche Orientierungsmuster und Regelwerke, verlieren traditionelle Strukturen und Institutionen ihre dominante Bedeutung, verschwimmen formale Konturen und Bezüge zu informellen und individuellen Beziehungsgeflechten. Der Begriff »Entgrenzung « macht nicht nur als (sozial-) wissenschaftliche Kategorie $^{1}$ Karriere, sondern dient auch in wirtschaftspraktischen Trendzeitschriften und in der öffentlichen Diskussion als feuilletonistische Metapher für aktuelle gesellschaftliche Entwicklungstendenzen (wie z.B. die »Entgrenzung des Büros« in Mensch und Büro 2001; die »Entgrenzung des Familienbegriffs« bei Sperber 2002).

Entgrenzung steht dabei für verschiedenste Auflösungsphänomene innerhalb tradierter Gesellschafts-, Wirtschafts- und Arbeitsstrukturen, die zum Teil als verkrustet und überholt, zum Teil aber auch als gesellschaftspolitisch notwendig eingeschätzt werden. Die Konsequenzen dieser Erosionsprozesse werden daher nicht selten einerseits als Errungenschaften einer fortschrittlicheren Moderne, andererseits zugleich als Verluste wertvoller Grundpfeiler des gesellschaftlichen Zusammenhalts eingestuft. Der inflationäre Gebrauch des Entgrenzungsbegriffs verwischt jedoch immer mehr das, worum es im Kern geht. Vor allem lenkt er davon ab, dass jeglichen Auflösungsprozessen selbst Grenzen gezogen sind (Minssen 2000; Boes u.a. 2001; Wolf/Mayer-Ahuja 2002) und dass sie wiederum neue Grenzziehungen und Strukturierungen mit sich bringen, mit denen sich neuartige strukturelle Muster oder institutionelle Formen von gesellschaftlichen Beziehungsgeflechten herauskristallisieren.

1) Eine Auswahl: »Entgrenzung von Organisationen « (Funder 2000), »Entgrenzung von Arbeit« (Kratzer 2003) bzw. »Entgrenzung von Kapital und Arbeit« (Döhl u.a. 2001), »entgrenzte Produktion« (Schmierl 2000), »Entgrenzung von Arbeit und Arbeitskraft« (Voß 1998), »Entgrenzung der Arbeit und der Arbeitszeit« (Wagner 2001) bzw. »entgrenzte Arbeitszeit« (Jurczyk/Voß 2000), »Entgrenzung von Arbeit und Leben« (Hielscher 2000), »Entgrenzung von Natur und Gesellschaft« (Lau u.a. 2002), »Entgrenzung des Lernens« (Böhle u.a. 2002, S. 110) und eben auch: »Entgrenzung betrieblicher Interessenvertretungen« (Brand1/Lawatsch 1999, S. 33). 
Diesen widersprüchlichen Effekten und Wechselwirkungen von Entgrenzung wird in diesem Beitrag mit Blick auf das deutsche System industrieller Beziehungen nachgegangen. Indem Erscheinungsformen der Auflösung und der Emergenz von Grenzziehungen in den Grundlagen und Strukturen inner- und überbetrieblicher Interessenvertretung der Arbeitnehmer gegenübergestellt werden, sollen Anhaltspunkte zur Beantwortung folgender Fragen gewonnen werden: Inwieweit wird durch Entgrenzungsprozesse das System industrieller Beziehungen aufgefächert zwischen traditionellen und stabilen, wenn auch tendenziell an Bedeutung verlierenden Formen einerseits und neuartigen, institutionell unterschiedlich verfassten Mustern der Interessenvertretung andererseits? Schreitet diese Auffächerung und Aufweichung der Strukturen immer weiter voran und treibt sie die industriellen Beziehungen zu einer intransparenten Vielfalt mit erheblichen Wirksamkeitsverlusten, oder bilden sich dabei implizit neue und andersartige Elemente heraus, die sich stabilisieren und zu einer (Re-) Vitalisierung der Interessenvertretung der Arbeitnehmer führen können? Inwieweit geht die Entwicklung dahin, dass eine klare Unterscheidung zwischen alten und neuen Formen nach dem Entweder-oder-Prinzip gar nicht mehr durchgängig möglich ist und dass neue Verschränkungen und Mischungsformen (»Sowohl-als-auch«) zu analysieren sind? Ziel des Beitrags ist es, in diesem komplexen und säkularen Prozess der Entgrenzung des Systems industrieller Beziehungen Anhaltspunkte und Voraussetzungen für mögliche, noch in statu nascendi befindliche Entwicklungsmuster herauszuarbeiten. ${ }^{2}$ Damit knüpfen wir an eine Kernüberzeugung der Theorie Reflexiver Modernisierung an, nämlich »dass Entgrenzung Entscheidung erzwingt: je mehr Entgrenzung, desto mehr Entscheidungszwänge, desto mehr provisorisch-moralische Grenzkonstruktionen, das heißt Grenzpolitik«(Beck/Bon//Lau 2004, S. 15; Hervorh. im Original).

\section{Entgrenzung - ein mehrschichtiger Begriff für verschiedenste Auflösungser- scheinungen}

Entgrenzung stellt einen mehrschichtigen Terminus dar, der, gerade weil er in vielfältiger Weise für unterschiedliche Auflösungserscheinungen verwendet wird, an Treffsicherheit verliert. In der generellen Debatte wird versucht, allgemein identifizierte oder interessenpolitisch jeweils befürwortete bzw. befürchtete Tendenzen des Zerfalls, der Aufweichung oder der Beseitigung bestehender wirtschaftlicher, arbeitsorganisatorischer, institutioneller und rechtlicher Strukturen und Regelungen als Entgrenzungsprozesse zu charakterisieren und nach den damit verbundenen Folgen für Akteure und Betroffene zu fragen. In diesem Zusammenhang stellt sich aber auch die Frage: Welche Bedeutung kommt der Tatsache zu, dass im Zuge solcher Entgrenzungsprozesse vielfach neue Grenzziehungen zu identifizieren

2) Mit diesem Beitrag wird weniger auf einen systematischen Überblick über unsere empirischen Forschungsarbeiten im Rahmen des Sonderforschungsbereichs 536 abgezielt (siehe dazu Schmierl u.a. 2001; Heidling u.a. 2004). Vielmehr soll es darum gehen, in selektiver und primär illustrativer Weise empirische Phänomene und Entwicklungsperspektiven im Feld industrieller Beziehungen im Hinblick auf die Grenzmetapher zu diskutieren. Darüber hinaus lassen sich die von uns festgehaltenen Trends in den industriellen Beziehungen als Beleg für die These anführen, dass sich die herkömmlichen Unterscheidungen der Ersten Moderne im Sinne eines »Entweder-oder « aufgrund gegenwärtig erkennbarer struktureller und institutioneller Veränderungen immer weniger aufrechterhalten lassen und zunehmend das Prinzip des »Sowohl-als-auch« leitend wird (Beck 2005). Es reicht demzufolge für eine angemessene Analyse des Wandels in diesem Feld nicht mehr aus, Reichweite und Wirkung von Interessenvertretung nach dem Entweder-oder-Prinzip zu unterscheiden: entweder auf betrieblicher Ebene oder außerhalb des Betriebes, entweder kollektive oder individuelle Interessenartikulation, entweder formalisierte Arbeitsregulierung oder komplette Regulierungslücke, entweder Betriebsratsrepräsentation oder Mitbestimmungslosigkeit, entweder nationale (d.h. deutsche) Unternehmensverfassung oder internationale, neoliberale Regelungswüste. Vielmehr müssen hier neue Verschränkungen und Mischungsformen analysiert werden. 
sind? Entgrenzungsprozesse zeigen sich in einer verstärkten Öffnung und Vervielfältigung bisher klar konturierter Strukturen und Scheidelinien, aber ebenso im Entstehen neuartiger Akteurskonstellationen sowie innovativer Organisations- und Institutionalisierungsformen, über deren Perspektive derzeit noch erhebliche Unsicherheit besteht.

In der Industrie- und Arbeitssoziologie wird der Begriff der Entgrenzung in der Bestandsaufnahme von seit längerem beobachtbaren Tendenzen zur Erosion von Normalarbeitsverhältnissen wie zur (Re-)Organisation von Unternehmensstrukturen, Arbeitsprozessen und Arbeitszeit verwendet (exemplarisch Kratzer 2003). Bekannte Grenzziehungen oder Grenzgefüge, durch die sich bislang bedeutsame gesellschaftliche, ökonomische oder rechtlichnormative Regulierungsmuster auszeichnen, büßen offenbar, was ihre Eignung zur eindeutigen Unterscheidung betrifft, an Bedeutung ein. Mit anderen Worten: »Diese Logik der Trennschärfe und Eindeutigkeit (der klassischen, industriegesellschaftlichen Hochmoderne, M.D./K.S.) stößt in der Gegenwart zunehmend an ihre Grenzen« (Beck u.a. 2004, S. 16). Die mit ihnen für die betroffenen Akteure verbundenen, bislang selbstverständlichen Prämissen, Absicherungen, Schutzwirkungen, Anspruchs- und Einflussmöglichkeiten verlieren an Stringenz und Zuverlässigkeit, weil sie sich aus ihrer bisherigen »Begrenztheit lösen « (Duden 2000, S. 341). Dadurch gehen Sicherheiten und Gewissheiten verloren und es öffnen sich Räume für Beliebigkeit oder gar Willkür, zumindest kommt es zu wachsenden Risiken und zu verstärkten Effekten der »Entsicherung « (vgl. etwa Moldaschl/Sauer 2000).

Grenzen und Grenzziehungen haben freilich auf allen gesellschaftlichen Ebenen seit jeher eine ambivalente Wirkungsweise: Einmal dienen sie dem Schutz vor und der Einschränkung von unerwünschten Belastungen, Einflüssen und Teilhaben durch andere gesellschaftliche oder ökonomische Interessengruppen bzw. Machtfaktoren; umgekehrt regeln und kanalisieren sie aber auch die Spielräume, die institutionell oder gesellschaftlich relevanten Akteuren (wie Unternehmen, unternehmerischen und nichtunternehmerischen Organisationen, Arbeitskräftegruppen, Anspruchsberechtigten, Verbänden, Institutionen etc.) für die Entfaltung politischer und ökonomischer Macht zugestanden werden. Durch Entgrenzungsprozesse werden solche Grenzziehungen erweitert, neu konfiguriert oder verschwinden völlig. Die Folge ist nicht nur, dass Schutzwirkungen und Teilhabeansprüche tendenziell verloren gehen bzw. die bisher begrenzten Einflussmöglichkeiten ausgeweitet werden; zugleich oder als Reflex darauf können sich Grenzziehungen verlagern oder in modifizierter Form (wieder) herausbilden, neuartige bzw. andersartige - mehr oder weniger institutionell basierte - Schutzoder Einflusselemente herauskristallisieren sowie veränderte Verhaltensmuster von Individuen und einzelnen Interessengruppen entwickeln (Beck u.a. 2004). Dadurch kann es zur Öffnung von Strukturen, zu einem neuen Gefüge von Grenzziehungen, aber auch zu innovativen Organisations- und Institutionalisierungskonstellationen kommen. Weil solche Entgrenzungsprozesse gleichzeitig grenzbildende Effekte oder Impulse mit sich bringen, umfasst für uns der Begriff der Entgrenzung immer beide Wirkungszusammenhänge, den des Auflösens bestehender ebenso wie den des Herausbildens neuer Grenzen. ${ }^{3}$ Dies scheint offenbar eine der Paradoxien des modernen Kapitalismus (Hartmann 2002) zu sein, die sich im Feld der industriellen Beziehungen in Form einer besonders vielfältigen Hybridisierung äußert (Schmierl 2001; Heidling u.a. 2004).

3) Mit diesem Verständnis von Entgrenzung sprengt der Begriff gleichsam seine eigene semantische Grenze, richtet er sich vom Sprachsinn her doch nur auf Prozesse und Effekte des Aufbrechens, Verschwindens oder Verlustes bereits bestehender institutioneller oder struktureller Grenzziehungen. 


\section{Entgrenzung als Erosions- und Innovationsmoment im System industrieller Beziehungen}

Mehrschichtigkeit und Ambivalenz von Entgrenzungsprozessen zeigen sich auf dem in diesem Beitrag analysierten Feld der industriellen Beziehungen in besonderer Weise. Zum einen zeichnet sich das deutsche System industrieller Beziehungen durch verschiedene Ebenen bzw. Arenen (vgl. Abel/Ittermann/Wannöffel 2001) der Interessenvertretung und -durchsetzung aus. Zum anderen handelt es sich um ein besonders verrechtlichtes und institutionell strukturiertes System (Schmierl 2001; 2003), das sich für die analytische Unterscheidung von Entgrenzung als Auflösung von Schutzgrenzen oder organisatorischen Scheidelinien einerseits und als Öffnung von Schranken für die Einflussnahme und Gestaltung von Interessenvertretung andererseits geradezu anbietet: Hier lassen sich zugleich parallele und gegenläufige bzw. kontinuierliche und diskontinuierliche Momente eines komplexen Entwicklungsprozesses betrachten, der bislang vor allem als Dezentralisierung, Flexibilisierung, Deregulierung etc. charakterisiert wurde. Gemeinhin werden diese Prozessbegriffe Tendenzen zugeschrieben, in deren Gefolge es generell zur Aufweichung und zum Verschwinden bisher selbstverständlicher Differenzierungen und Abgrenzungen - von Schutzzäunen ebenso wie von Einflussbeschränkungen - kommt, mit all ihren ambivalenten Effekten: einerseits einem neuartigen Ausgeliefertsein der davon betroffenen Akteure gegenüber einer Schrankenlosigkeit von Machteinflüssen anderer, andererseits damit verbundenen emanzipatorischen Momenten, neuen Möglichkeiten, individuell und kollektiv größere Freiheitsgrade zu nutzen, d.h. sich persönlich freier zu entfalten.

Dementsprechend äußert sich die mit dem Begriff der Entgrenzung erfasste Entwicklung der Interessenvertretung der Arbeitnehmer nicht nur darin, dass Grenzziehungen der Organisation, der Regelungsreichweite und der Verhandlungsfelder von Arbeitnehmervertretungen zunehmend durchlässig werden und damit selbstverständliche Sicherungen, Mitwirkungsansprüche etc. an Bedeutung verlieren. Es werden zugleich auch Grenzziehungen von den Akteuren gezielt verändert oder aufgegeben, um sie durch neue, ggf. weiter gezogene Grenzlinien und neuartige Einflusspotentiale zu ersetzen. Damit können sie möglicherweise den negativen Effekten bisheriger Entgrenzungstendenzen auf anderen Ebenen entgegenwirken oder Innovationen erreichen, durch die sich qualitativ neue Schutz- oder Gestaltungspotenziale eröffnen und das bestehende System der industriellen Beziehungen eine Bereicherung erfährt.

Entgrenzung bedeutet daher auch auf diesem Feld nicht einfach die bloße Erosion oder Auflösung von Grenzen. Zum einen stößt sie früher oder später selbst auf prozessimmanente Grenzen aufgrund der damit verbundenen Risiken sowohl für die Unternehmen als auch für die Arbeitnehmer (Moldaschl/Sauer 2000; Kratzer 2003; 2005). Zum anderen zeigen sich solche Grenzen auch in neuartigen Koordinations- und Kooperationsschwierigkeiten, deren Überwindung wiederum nach neuartigen Zuordnungen und Verarbeitungsprozeduren verlangt (Funder 2000). Vor allem aber werden im Zuge solcher Entgrenzungsprozesse bisherige Grenzziehungen vervielfältigt, modifiziert, substituiert oder auf ein qualitativ neues Niveau gehoben, über dessen Relevanz für die Betroffenen wie für die außenstehenden Beobachter bislang noch erhebliche Unklarheit besteht. Hinzu kommt, dass in vielen Bereichen des Systems industrieller Beziehungen herkömmliche Grenzziehungen nach wie vor strukturelle Wirkung entfalten bzw. verhaltensprägend bleiben, sodass traditionelle und innovative Strukturen bzw. Grenzziehungen einschließlich neuer Offenheiten nebeneinander (weiter) bestehen. Entgrenzungsprozesse in den industriellen Beziehungen umfassen also Erosionsund Re- bzw. Neustrukturierungsvorgänge, die zeitgleich oder zeitversetzt, völlig separat oder in struktureller Verschränkung ablaufen können.

Im Folgenden wollen wir den entgrenzungsbedingten Entwicklungen in den Grundlagen einer inner- und überbetrieblichen Interessenvertretung nachgehen, wie sie sich im Rahmen 
unserer konzeptionellen und empirischen Studien darstellen, und daraus Schlussfolgerungen zur Bedeutung dieser Veränderungen für die Gesamtheit des deutschen Systems industrieller Beziehungen ziehen. Dazu werden zunächst die bedeutsamsten Veränderungen skizziert, wie sie im Zuge der seit längerem stattfindenden Auflösung betrieblicher Außen- und Binnengrenzen zu beobachten sind, um dann auf den damit verbundenen Wandel in den Grenzziehungen des Systems der industriellen Beziehungen näher einzugehen.

\section{Erosions- und Restrukturierungseffekte durch Auflösung betrieblicher Außen- und Binnengrenzen}

Mit den in zahlreichen Untersuchungen konstatierten und analysierten Prozessen der sukzessiven Auflösung der Außen- und Binnengrenzen von Unternehmen und Betrieben verbinden sich gravierende Veränderungen in den Grundlagen und Möglichkeiten der Interessenvertretung von Arbeitnehmern. Es lassen sich vor allem Erosionseffekte, aber auch Impulse für neue Modi und Konstellationen der Interessenvertretung erkennen (Schmierl u.a. 2001).

\subsection{Effekte durch die Aufösung betrieblicher Außengrenzen}

Die Auflösung der Außengrenzen von Betrieben vollzieht sich als Folge von Internationalisierungs- bzw. Globalisierungsprozessen und der Restrukturierung von Wertschöpfungsketten mit grundlegenden Konsequenzen für die Formen und Potenziale von Interessenvertretung. Die Globalisierung der Weltwirtschaft und die Verlagerung von Betrieben im Zuge der Internationalisierung von Produktions- und Dienstleistungsaktivitäten führen zu mehr oder weniger gravierenden Beeinträchtigungen von Betriebsratshandeln und Gewerkschaftspolitik. Vor allem zeigt sich, dass die Strukturen der nationalstaatlich geprägten industriellen Beziehungen, die sich in Deutschland in ihren Zuständigkeits- und Einflussbereichen vorrangig an den Außengrenzen von Betrieben und Unternehmen oder an regionalen bzw. branchenbezogenen Tarifbereichen ausrichten, immer weniger adäquat werden. Denn innerhalb transnationaler Produktionsverbünde (und aufgrund von Produktionsverlagerungen ins Ausland) werden die Entscheidungsstrukturen auf der Ebene des Einzelbetriebs intransparent und in überbetrieblicher Perspektive Arbeits- und Mitwirkungsstandards heterogen. Aber auch die eng damit verknüpfte Neuordnung der Wertschöpfungsprozesse (durch Outsourcing, Aufspaltung und Zusammenschlüsse von Unternehmen, Dezentralisierung, Konzentration auf Kernkompetenzen oder Portfoliomanagement) führt zu problematischen Auswirkungen nicht nur für die davon betroffenen Betriebe, sondern vor allem für deren Interessenvertretungen, die in ihrem Einfluss- und Verhandlungspotenzial entscheidend geschwächt werden, geht ihnen doch vielfach der Verhandlungsadressat im eigenen Betrieb verloren (Deiß 2000). Aufgrund solcher Entwicklungen sinkt der Einfluss der betrieblichen Interessenvertretung etwa auf Standortentscheidungen, auf die Fragmentierung der Betriebsratsgremien oder hinsichtlich der Möglichkeiten kooperativer Konfliktbewältigung (vgl. Funder 2000; Stumpf-Fekete 2000; Abel/ Sperling 2001).

Innerhalb der bestehenden dualen Interessenvertretungsstrukturen entwickeln sich Vernetzungsansätze, die sich der Entgrenzung der betrieblichen Interessenvertretung entgegenstellen. So werden etwa in Reaktion auf die fortschreitende Zersplitterung von Großunternehmen in zahlreiche selbstständige Betriebseinheiten sog. Standortbetriebsräte gebildet. Diese ermöglichen auch für die Beschäftigten von an- bzw. ausgegliederten, wenn auch formal selbständigen Betrieben, die auf dem Betriebsgelände eines dominanten (Abnehmer-)Unternehmens angesiedelt sind, zumindest eine partielle - wenn auch nur vermittelt über dessen Betriebsrat wirkende - Interessenvertretung (vgl. auch Gesterkamp 2000; Wassermann 2002).

Noch selten - wenn auch mit zunehmender Tendenz - werden Betriebsrätenetzwerke eingerichtet, mit deren Hilfe der durch die Entgrenzung der Unternehmen bedingten informato- 
rischen und wissensbezogenen Zersplitterung der Interessenvertretung entgegenzuwirken versucht wird (vgl. Deiß/Heidling 2000). Ähnliche Effekte werden mit Hilfe von Netzwerken außerbetrieblicher Gewerkschaftsarbeit oder von regionalen Entwicklungsagenturen unter Beteiligung von Betriebsräten und Gewerkschaften zu erreichen versucht. Schließlich werden weiter reichende Konzepte angedacht, wie eine vernetzte Interessenvertretung entlang von Wertschöpfungsketten oder die Realisierung von Netzwerkgewerkschaften bzw. wie Ansätze zur betrieblichen Mitbestimmung und Arbeitnehmerbeteiligung innerhalb von Unternehmensnetzwerken auszusehen hätten (vgl. Sydow/Wirth 1999; Wehling 2000; Martens 2000). ${ }^{4}$

Ein weiterer Aspekt der Auflösung betrieblicher Außengrenzen kommt in zwei Tendenzen zur vermehrten Herausbildung von Kleinbetrieben zum Ausdruck: Einerseits werden im Zuge von Fragmentierungs- und Dezentralisierungstendenzen herkömmliche Unternehmensabteilungen immer häufiger zu formal eigenverantwortlichen, wenn auch auftrags- und prozessbezogen weiterhin stark abhängigen kleinen betrieblichen Einheiten verselbstständigt; andererseits entstehen im expandierenden Dienstleistungs- und Servicesektor zunehmend eigenständige Klein- und Kleinstunternehmen (Schmierl 2001; Bosch u.a. 2002). Beides hat erhebliche Folgen für die Interessenvertretung im Sinne von »Mitbestimmungsdilemmata (Schmierl 2003b): Insgesamt gehen die Betriebsratsgründungen zurück ${ }^{5}$, gleichzeitig nehmen die Schwierigkeiten bei der Organisierung einer betrieblichen Interessenvertretung zu (vgl. Ellguth 2004; Benz-Overhage 2000). Der säkulare Trend zu tertiären Wirtschaftssegmenten und kleineren Wirtschaftseinheiten schränkt zunehmend den Bereich der Ökonomie ein, in dem im historischen Rückblick in Deutschland die am weitesten gehenden Mitbestimmungsrechte erkämpft wurden; ablesbar am Beispiel der schwindenden Geltungsbereiche der Montanmitbestimmung (vgl. Lompe u.a. 2003).

Veränderungen der Interessenvertretung sind schließlich im Gefolge der Reorganisation von Unternehmensstrukturen durch Shareholder-Value-Orientierungen zu konstatieren (Meil/ Heidling/Schmierl 2003; Höpner 2003; Streeck/Höpner 2003).

Im Zusammenhang mit Strategien einer kapitalmarktorientierten Unternehmensführung werden Grenzverschiebungen gewissermaßen zum Prinzip erhoben. In einem von uns empirisch untersuchten, global agierenden deutschen Konzern der Elektroindustrie (Meil/ Heidling/Schmierl 2003) zielen die Programmvorgaben zur Shareholder-Value-Orientierung auf eine konsequentere Orientierung am Geschäftserfolg, die Optimierung des Geschäftsportfolios und die Verbesserung der Kapitalstruktur. Als Konvergenz sichernder Maßstab für die Leistungsbewertung der Unternehmensstandorte dient eine für alle Bereiche verbindliche Kenngröße zur Ermittlung des Unternehmenswertes und -erfolgs, deren Erreichung durch die einzelnen Standorte Konzernentscheidungen in Richtung »buy«, »cooperate«, »sell« oder »close« scheinbar mathematisch steuert. Personalpolitisch folgenreiche Vorgaben, für die im deutschen Betriebsverfassungsgesetz verbindliche Verhandlungsregeln unter Beteiligung der Betriebsräte definiert sind, werden über eine derartige Portfolio-Politik, bei der durch Outsourcing, Produktionsverlagerung und Fremdvergabe (unter der Bezeichnung »Desinvestitionen«) Unternehmensstrukturen und Abteilungsgliederungen ständig neu geschnitten werden, allenfalls indirekt und vermittelt thematisiert und aus der mitbestimmten Verhandlungsroutine eskamotiert. Die auf den Arbeitsplatz begrenzte Regulierung, aus der die Betriebsräte bisher ihre Aushandlungsmacht ableiten konnten, wird

4) Vor dem Hintergrund verschwimmender Grenzen zwischen Unternehmen und Umwelt infolge der Dezentralisierung von Organisationen und der Auflösung der Unternehmensgrenzen wird sogar die Frage nach dem »Ende kollektiver Interessenvertretung « (Kühl 1998) aufgeworfen.

5) Trotz Hinweisen auf gegenläufige Tendenzen im Dienstleistungsbereich bei Trautwein-Kalms 2001. 
zunehmend durch internationale Restrukturierungen ersetzt. Mit diesen gewandelten Management-, Steuerungs- und Herrschaftsprinzipien sind die traditionellen Formen, Methoden und Routinen einer betrieblichen und überbetrieblichen Interessenvertretung nicht mehr kompatibel und bleiben folglich unwirksam. Zwar werden die nationalen gesetzlichen und (tarif-) rechtlichen Bedingungen durch das Management nicht zielgerichtet umgangen, doch wird den betriebsverfassungsgesetzlichen Bestimmungen aufgrund ihrer nationalen und betrieblichen Begrenztheit der Anwendungsbereich entzogen. Die primär nationalstaatlich begrenzten Strukturen von industriellen Beziehungen werden umso mehr inadäquat, als personalpolitische Maßnahmen, weil sie auf internationale Portfolio-Entscheidungen zurückgehen, von den nationalen Systemen der Arbeitsregulierung entkoppelt werden. Zudem steigt für die Interessenvertretung der Informationsbedarf zu ökonomischen Zusammenhängen, Eigentumsregelungen und wechselseitigen Verrechnungsmethoden im multinationalen Konzern, für deren Bewertung allerdings ihre Kompetenzen nicht ausreichen.

Offensichtlich wird dabei vor allem, dass aufgrund der zunehmenden Entkoppelung von Finanz- und Realkapital und der wachsenden Bedeutung des Shareholder Value im konkreten Betriebsalltag für die einzelnen Betriebe in den Produktions- und Unternehmensverbünden Prämissen hinsichtlich Personalrelationen, Kostendeckungsbeiträgen, Ertragsgrößen usw. gesetzt werden, mit denen sich die traditionellen Interessenvertretungen kaum mehr auseinander setzen können, ist doch auch das lokale Management selbst diesen gegenüber tendenziell hilflos. Allerdings eröffnen sich für die Interessenvertretungen mit solchen Entwicklungen vereinzelt zugleich auch Optionen, ihren Einfluss für verschiedene internationale Standorte zur Geltung zu bringen und zu standortübergreifenden Formen der Interessendurchsetzung zu gelangen. Dazu bedarf es freilich des Aufbaus geeigneter Kompetenzen bei den Betriebsräten vor Ort und der Entwicklung betriebsübergreifender Verhandlungsstrukturen, Voraussetzungen, die auch für die Bildung von effizienten Euro-Betriebsräten als notwendig erachtet werden (vgl. dazu Deiß/Mendius 2005). Mit der Nutzung derartiger Optionen können sich grundlegende Grenzverschiebungen ergeben, indem z.B. Betriebsräte gegenüber den Unternehmenszentralen den Schulterschluss mit dem in seiner Entscheidungshoheit geschwächten Standortmanagement suchen (Deiß/Heidling 2001; Meil/Heidling/Schmierl 2003).

\subsection{Effekte durch die Auflösung betrieblicher Binnengrenzen}

Auflösungstendenzen der Binnengrenzen von Betrieben zeigen sich vor allem im Zuge arbeitskraftbezogener Rationalisierungsstrategien und der damit verbundenen Internalisierung von Marktprinzipien in die Binnenstruktur der Betriebe als inhaltliche, zeitliche und soziale Entgrenzung von Arbeit (Döhl u.a. 2001). Solche Entwicklungen finden insbesondere im Rahmen der breiten Einführung verschiedener Formen von Gruppenarbeit und selbstverantwortlicher Leistungserbringung statt, was wiederum gravierende Veränderungen in der bisherigen Vertretungsarbeit nach sich zieht: in Richtung forcierter Betreuungstätigkeit sowie erhöhter diskursiver Koordinierungsarbeit hinsichtlich individualisierter Gruppensprecher (Gerst 2004), in Richtung verstärkter gestaltungsorientierter Projektarbeit (Martens 2001) und in Richtung einer intensiveren »Verbetrieblichung « der Arbeitsregulierung, indem ein Zuwachs an Funktionen und Kompetenzen für die Betriebsräte konstatiert wird (MüllerJentsch 2000).

In solchen Veränderungen kommt allerdings nicht nur die Erosion bisheriger Grenzziehungen im System industrieller Beziehungen zum Ausdruck. Es verbindet sich damit auch eine Vervielfältigung tariflicher und betrieblicher Festlegungen (z.B. aufgrund von Haustarifverträgen, betrieblichen Bündnissen für Arbeit, individuellen Zielvereinbarungen etc.) und eine Heterogenisierung bisher vergleichsweise gleichartiger Verhandlungs- und Entschei- 
dungsverfahren auf der Interessenvertretungsebene. Damit kommt es vor allem zu einer Öffnung bislang selbstverständlicher Grenzlinien der Regulierung von Beschäftigungs- und Arbeitsaspekten: Traditionell auf überbetrieblicher bzw. tariflicher Ebene fixierte Arbeitsstandards werden in wachsendem Maße auf einzelbetrieblicher Ebene unterschiedlich verhandelt, sodass für die Betriebsbelegschaften ähnlich strukturierter Branchensegmente immer häufiger heterogene Arbeitsstandards gelten (Deiß 2000).

Insbesondere die in den 90er-Jahren angesichts zunehmender Krisensituationen in zahlreichen Unternehmen - oft abweichend von den tariflichen Regelungen - eingerichteten betrieblichen Bündnisse für Arbeit tragen zu dieser Heterogenität bei (Rehder 2002; Seifert 2002). Auch wenn dabei bislang dem Weisungsrecht der Geschäftsleitung oder der tariflichen Regelung unterliegende Sachverhalte zum Gegenstand solcher Modernisierungspakte auf betrieblicher Ebene werden, scheint es fraglich, ob angesichts der tendenziell schwächeren Position der Betriebsräte insbesondere in den Krisensituationen, in denen solche Bündnisse geschlossen werden, von einer »schleichenden Erweiterung der betrieblichen Mitbestimmung « (Rehder 2003) gesprochen werden kann. Denn abgesehen davon, dass diese Entwicklung zu Lasten des ohnehin an Breitenwirksamkeit verlierenden Flächentarifs geht, können die dadurch eröffneten Möglichkeiten der Betriebsräte zur Mitgestaltung kaum den drohenden (oder bereits eingetretenen) Verlust gewerkschaftlicher Gestaltungskraft ausgleichen (Altmann/Deiß 1996). Zudem besteht für derartige betriebliche Bündnisse für Arbeit immer auch das Risiko, wegen negativer ökonomischer Rahmenbedingungen zu scheitern bzw. wegen veränderter Management- oder Eigentümerstrukturen kurzfristig revidiert zu werden (Deiß 2000), mit der unmittelbaren Folge des Verlustes solcher nur auf betrieblicher Ebene eingeräumter Mitwirkungsmöglichkeiten.

Auch im Zusammenhang mit der Einführung neuer Entgeltsysteme ist in zunehmendem Maße eine Verlagerung der Aushandlung von variablen Lohn- und Leistungsbestandteilen auf die Ebene von Arbeitsteams oder Beschäftigten festzustellen (Schmierl 1995; Bender 1997). Die Auflösung bisher gültiger Binnengrenzen lässt sich aber auch im Hinblick auf veränderte Formen der Arbeitszeitgestaltung ausmachen (Steinrücke u.a. 2001; SchulzeBuschoff 2000); hier zeichnet sich ebenfalls ein Trend zur Verschiebung der Regulierung der Arbeitszeiten von der Tarif- auf die Betriebsebene oder gar zur Individualisierung der Arbeitszeitregelung ab, weshalb auch von einer Verbetrieblichung der Arbeitszeitpolitik (Hielscher 2000) gesprochen werden kann.

Wir sehen in solchen Tendenzen zwar ebenfalls eine schleichende Verbetrieblichung der deutschen industriellen Beziehungen. Allerdings zeigen unsere empirischen Ergebnisse, dass diese Verbetrieblichung weniger zu einer Verschiebung des Machtpotenzials von den Gewerkschaften zu den einzelbetrieblichen Interessenvertretungen führt; vielmehr gewinnen mit der Entgrenzung von Arbeit und Beschäftigungsverhältnissen arbeitnehmerrelevante Handlungsfelder (etwa der Weiterbildung und der Arbeitsorganisation) an Bedeutung, die schwerlich auf tarifpolitischer Ebene standardisiert und dort verbindlich festgelegt werden können. Diese Handlungsfelder wachsen nunmehr den Betriebsräten zu, die in ihrer Verhandlungsmacht und in ihrer institutionellen Wirksamkeit in der Regel aber eine weit schwächere Position als die Gewerkschaften aufweisen.

Nicht zuletzt werden organisatorische und arbeitskräftegruppenbezogene Grenzziehungen in den Binnenstrukturen durchlässiger oder zum Verschwinden gebracht, wenn neue Managementkonzepte umgesetzt werden, die sich an neuen Leitbildern wie etwa dem des »Arbeitskraft-Unternehmers« orientieren (Pongratz/Voß 2003; 2004; Eichmann 2004). Gleichzeitig jedoch entstehen neben den oder anstelle der formalisierten Interessenvertretungsformen neue Ansatzpunkte einer direkten Partizipation, die auch ohne Beteiligung der Betriebsräte jenseits bisheriger Grenzziehungen eine wirksame individuelle Interessenvertre- 
tung zu erlauben beanspruchen (Abel u.a. 2001; Schmierl 2005). Dabei ist die Frage nicht leicht zu beantworten, ob und inwiefern entsprechende Ansätze bestehende Interessenvertretungsstrukturen schwächen oder ergänzen - oder ob und inwieweit sie zu völlig neuen Arrangements führen.

\section{Nicht nur Erosion, sondern vor allem auch veränderte und neue Grenzzie- hungen im System industrieller Beziehungen}

Die bislang aufgeführten Veränderungen in Struktur und Praxis der Vertretung von Arbeitnehmerinteressen zeigen zumindest mit Blick auf Deutschland deutlich: Mit der Erosion und der Restrukturierung der Interessenvertretungsstrukturen verbinden sich Prozesse des Wandels in den Grenzziehungen des Systems industrieller Beziehungen selbst, die sich auf verschiedenen Ebenen dieses Systems abspielen. Zum einen kommt es durch die Entinstitutionalisierung traditioneller Interessenvertretungsstrukturen $\mathrm{zu}$ einer Vervielfältigung und Öffnung und damit zur Auflösung bisher selbstverständlicher Grenzziehungen, zum anderen aber auch - u.a. als Reflex darauf - zu Grenzüberschreitungen und zur Herausbildung neuer Grenzlinien und schließlich zur Emergenz neuartiger, durch mehr oder weniger (re-)institutionalisierte Grenzziehungen verfestigter Formen und Muster von Interessenvertretung.

\subsection{Pluralisierung und Öffnung bisheriger Grenzziehungen}

Die Tendenz zur Erosion grundlegender Elemente des klassischen deutschen Systems der industriellen Beziehungen kommt weniger in einem einfachen Wegfall bisher institutionell vorgegebener bzw. strukturell dominanter Grenzziehungen zum Ausdruck als vielmehr in ihrer sukzessiven Pluralisierung und Öffnung. Durch fortschreitende Internationalisierung, Unternehmenszersplitterung, globale Produktionsnetzwerke und Dezentralisierung von Entscheidungs- und Arbeitsstrukturen werden Interessenvertretungszuständigkeiten und arbeitnehmerrelevante Festlegungen vervielfältigt und aufgeweicht (Schmierl/Pfeiffer 2005). Herkömmliche Interessenvertretungen verlieren mit der Zersplitterung von Großunternehmen in rechtlich selbstständige, aber in der Wertschöpfungskette stark abhängige Klein- und Mittelunternehmen (KMU) ihre traditionellen Verhandlungsadressaten (Deiß 2000). Zudem hat die Verbandsflucht von Unternehmen deutlich zugenommen (z.B. ist 2001 die Tarifbindung ostdeutscher Unternehmen auf $15 \%$ zurückgegangen); zahlreiche Unternehmen treten sog. OT-Verbänden bei, die zwar Teil herkömmlicher Arbeitgeberverbände, jedoch ohne tarifliche Bindung sind. Immer mehr Betriebe weichen also offiziell oder nichtoffiziell von tariflichen Bestimmungen ab (durch Nutzung von Öffnungsklauseln, durch die eben angesprochenen Bündnisse für Arbeit, aber auch durch Unterlaufen von Regelungen) und vereinbaren einzelbetriebliche oder gar gruppen- oder personenindividuelle Regeln, sodass bislang homogene und verbindliche Lohn- und Arbeitsstandards verstärkt heterogenisiert werden (Ellguth/Kohaut 2004; Kohaut/Schnabel 2003; Artus 2004; Bispinck 2004; Deiß/ Mendius 2005).

Im Zuge dieser Entwicklung wird nicht nur das tradierte System industrieller Beziehungen entinstitutionalisiert und die Reichweite des Flächentarifvertrags von Mal zu Mal verringert (so auch Rehder 2003). Auch die kollektive Interessenvertretung selbst nimmt zunehmend heterogene Formen an. Innerhalb der dualen Interessenvertretungsstruktur wird die über die Organisation vermittelte, wechselseitige solidarische Absicherung ihrer Verhandlungsstärke geschwächt und die Entwicklung gemeinsamer interessenpolitischer Positionen zusehends erschwert. Das Gros der Betriebsräte bleibt dabei ihrer traditionell passiven und reaktiven Orientierung zum Schutz der Arbeitnehmer verhaftet.

Insgesamt wird deutlich: Derartige Veränderungen in den Grenzziehungen stellen die duale Interessenvertretung als eine der Basisinstitutionen des deutschen Systems industrieller Bezie- 
hungen in Frage, vervielfältigen sich doch auch die hierunter zu fassenden Formen und Muster in Arbeitsteilung und Zusammenwirken der Interessenvertretungen auf einzel- und überbetrieblicher Ebene.

\subsection{Herausbildung neuartiger Grenzlinien}

Allerdings führen die skizzierten Öffnungstendenzen auch zur Überbrückung bislang selbstverständlicher Zuständigkeits- und Hierarchiestrukturen innerhalb dieser Dualität. Durch solche Grenzüberschreitungen, aber auch im Zuge produktions- und branchenökonomischer Veränderungen kommt es zu neuartigen Grenzlinien der Interessenvertretung, die sich z.T. in Ergänzung, z.T. in Abhebung zu den weiterhin bestehenden herkömmlichen Strukturen herausbilden. Neue organisationale, klientelbezogene und inhaltliche Grenzziehungen zeichnen sich vielfach dadurch aus, dass sie offener, flexibler und reversibler gehandhabt werden, als dies in den Verhandlungsbeziehungen und Aushandlungsaktivitäten der traditionellen Interessenvertretung der Fall bzw. überhaupt möglich ist.

Deutlich wird dies etwa dann, wenn gewerkschaftliche Abteilungen etwa der Betriebs-, Bildungs- und Tarifpolitik, die bislang durch strikte Aufgabenabgrenzungen und Abschottungen geprägt waren, gemeinsame Projekte der Organisations- und Netzwerkentwicklung betreiben und miteinander verwobene Vorgehensweisen praktizieren oder wenn sie jenseits tradierter Funktionsschneidungen neue, miteinander verknüpfte Qualifizierungs- und Beratungsangebote einrichten (Deiß/Heidling 2001). Ähnliches gilt, wenn bislang betriebsfokussiert agierende Betriebsräte und überbetrieblich orientierte Gewerkschaftsfunktionäre im Rahmen von Betriebsrätenetzwerken die Grenzen ihrer Zusammenarbeit neu definieren.

Besonders augenscheinlich werden Herausbildungen neuer Grenzlinien dann, wenn sie sich als organisatorische bzw. institutionelle Restrukturierungen äußern. In besonderem Maße trifft dies auf Mergers\&Acquisitions von Gewerkschaftsorganisationen zu, wenn z.B. kleinere Gewerkschaften in und durch größere integriert werden oder wenn sich Einzelgewerkschaften zusammenschließen. Insbesondere mit der Fusionierung der Einzelgewerkschaften IG Medien, HBV, DPG, DAG und ÖTV zu ver.di suchten die im Vertretungsbereich von Dienstleistungstätigkeiten agierenden Gewerkschaften den zunehmend entgrenzten Branchen- und Unternehmensstrukturen in diesem Wirtschaftsfeld und den damit einhergehenden Schwächungen der Arbeitnehmervertretungen durch weitreichende institutionelle Reorganisationsmaßnahmen zu begegnen. Ähnliche Zielsetzungen verfolgte die IG Metall mit der Integration der Gewerkschaft Holz und Kunststoff bzw. der Gewerkschaft Textil und Bekleidung (vgl. Keller 2004; Hassel 2000; Schmierl 2003a).

Neuartige Grenzziehungen werden aber auch mit Blick auf gegenwärtig diskutierte Phänomene der Neuschneidung gewerkschaftlicher Mitgliederstrukturen entlang von Berufsgruppen bzw. von Beschäftigten mit begehrten und weniger begehrten Qualifikationen diskutiert und erprobt (als Beispiele seien hier die Pilotenvereinigung Cockpit und die Unabhängige Organisation der Flugbegleiter genannt). Während es hier um die Bildung neuer Interessenvertretungsakteure auf Basis berufsbezogener Grenzziehungen innerhalb bisheriger Klientelstrukturen geht, finden sich andere Experimente, mit denen versucht wird, herkömmliche Schranken gegenüber anderen Klientelgruppen so zu öffnen, dass diese für traditionelle Interessenvertretungsformen wie Gewerkschaft oder Betriebsrat innerhalb neuartiger, gewissermaßen organisationsunabhängiger Beteiligungs- und Betreuungsformen gewonnen werden können (vgl. Satzer 2001).

Nicht zuletzt werden in Fällen, in denen Arbeitnehmervertreter aktive und gestaltungsorientierte Politik machen, die Grenzen betrieblicher Interessenvertretung überschritten bzw. weiter gesteckt, als dies bislang üblich und institutionell abgesichert ist; dies geschieht insbesondere dann, wenn Betriebsräte offensiv und mit eigenen Konzepten versuchen, selbst 
strategisch auf Umstrukturierungsmaßnahmen und Entscheidungen des Managements zur Unternehmensentwicklung und Standortsicherung im Interesse der Beschäftigten Einfluss zu nehmen, Aktivitäten, die freilich nur möglich sind, wenn die Betriebsräte über geeignetes Expertenwissen verfügen bzw. dieses umfassend erschließen können (vgl. Deiß/Heidling 2001). ${ }^{6}$

\subsection{Emergenz neuer institutioneller Formen und Muster der Interessenvertretung}

Darüber hinaus emergieren im Verlauf der Erosion bisheriger und der Herausbildung neuer Grenzziehungen innovative, mehr oder weniger institutionalisierte Formen und Muster von Interessenvertretung. Dieser Prozess reicht von der zunächst eher informellen Verfestigung neuartiger Interessengruppierungen und Verhandlungskonzepte bis hin zu ihrer institutionellen Verfassung als völlig neue Akteurs- und Organisationskonstellationen. Handlungsmuster und Strukturen solch neuer Formen der Interessenvertretung orientieren sich dabei zum Teil an den Vorbildern des traditionellen Systems industrieller Beziehungen, weisen aber durchaus auch neue Elemente einer flexiblen Interessenvertretung auf.

Neben den bereits erwähnten Beispielen der Bildung sog. Standortbetriebsräte oder betriebsratsähnlicher Gremien oder Akteure in KMU sind neue Muster der kollektiven Interessenvertretung in der dauerhaften Etablierung von Betriebsrätenetzwerken ebenso auszumachen wie in den bislang sehr informellen, kaum institutionalisierten Netzwerken außerbetrieblicher Gewerkschaftsarbeit: In ihnen werden auch arbeitslose und verrentete Arbeitnehmer zu Akteuren kollektiver Interessenvertretung und damit zu einem - wenn auch noch unbedeutenden - Bestandteil des Systems industrieller Beziehungen. Weitere neuartige Formen der Interessenvertretung stellen Entwicklungsagenturen dar, in denen Betriebsräte und Gewerkschaften gemeinsam mit regionalen und kommunalen Stellen sowie wirtschafts- und arbeitsmarktpolitisch relevanten Akteuren versuchen, jenseits bisheriger Scheidelinien Arbeitnehmerinteressen in einen regionalpolitischen Problemlösungsprozess einzubringen und die Interessenauseinandersetzung einer multilateralen und reflexiven Entscheidungsfindung zuzuführen.

Deutlich werden damit Ansätze zu kollektiven Strategien, die nicht eingegrenzt sind auf die traditionellen Akteure industrieller Beziehungen sowie die klassischen Verhandlungsfelder und Verhandlungsgegenstände. Ausschließlich nationale Verhandlungsprozeduren werden zwar nicht unwichtig, verlieren jedoch insbesondere in Großbetrieben und großen Mittelstandsunternehmen ihre bisherige Stellung als die zentrale Macht- und Legitimationsbasis für die Akteure der industriellen Beziehungen. Die den internationalen Kapitalstrategien adäquaten Aushandlungsarenen verlagern sich zusehends von der ursprünglich nationalen bzw. regionalen Ebene der Tarifverhandlungen (auf der Tarifvertragsebene) bzw. Betriebsvereinbarungen (auf der Betriebsebene) in zwei neue Arenen mit jeweils veränderten Verhandlungsthemen, Legitimationsgrundlagen und Vereinbarungsformalismen. So findet verstärkt eine Mehrgleisigkeit der Verhandlung zu klassischen arbeitspolitischen Themen statt: auf einer transnationalen Ebene (z.B. durch Europäische Tarifverhandlungen, grenzüberschreitende Gewerkschafts- und Arbeitgeberverbands-Kooperationen, Europäische Betriebsräte), auf der regionalen Ebene (z.B. durch Teilnahme an Regionalkonferenzen, Runden Tischen, Agenda-21-Gruppen o.ä.) und zugleich auf einer politischen Ebene (durch die Entwicklung von Lösungen zur Wirtschafts-, Innovations-, Standort- und Regionalpolitik) (vgl. Beese/ Dörre/Röttger 2004; Deiß/Mendius 2005). In beiden Arenen gewinnen neue Themen und

6) Dass in diesem Kontext die Mitwirkungsmöglichkeiten einzelner Betriebsräte mittlerweile thematisch eine Erweiterung erfahren haben, wird im Rahmen der gegenwärtigen Diskussion um eine weitere Entgrenzung und Pluralisierung der Unternehmensmitbestimmung (etwa zwischen Großkonzernen und mittelständischen Unternehmen) inzwischen von Arbeitgeberseite explizit zum Gegenstand der Kritik gemacht (Viering 2004). 
Akteurskonstellationen insofern an Bedeutung, als relevante Steuerungsleistungen für die Schaffung, Sicherung und Reproduktion von Beschäftigungsverhältnissen in einer Region z.B. von einer Standortpolitik gemeinsam mit kommunalpolitischen Verwaltungen oder - in Europa - von Kontakten zu politischen Parteien im Europäischen Parlament ausgehen.

Ferner entwickeln auch traditionelle Interessenvertretungsakteure ein verändertes Rollenverständnis und andere Formen der Betriebsrats- und Gewerkschaftsarbeit, wenn neue Arbeitsweisen wie Projektmanagement und Projektarbeit anstelle von Ausschussarbeit, neue Veranstaltungsformen wie dialog- und diskursorientierte Betriebsversammlungen, internetbasierte Plattformen zum Austausch und zur Diskussion von Erfahrungen und Erwartungen Einzug halten (vgl. Martens 2001). Solche Neuerungen zeigen sich nicht nur in einzelnen Betrieben des »Alten Marktes«, also der Produktionsindustrie und des Handels, sondern vor allem in den Unternehmen der neuen Wissens- und Dienstleistungsökonomie. So kann in dem von ver.di initiierten Projekt connexx.av als einem neuartigen und eher informellen Versuch von Interessenvertretung zugleich ein funktionales Äquivalent zur herkömmlichen Gewerkschaftsarbeit im Dienstleistungsbereich gesehen werden: Gewerkschaftsarbeit nähert sich dadurch der in solchen Unternehmen vorherrschenden individualisierten Denk- und Lebenswelt an und mutiert selbst zur Dienstleistung für Einzelpersonen in Form von individuellen Beratungsangeboten in arbeitsrechtlichen Angelegenheiten, für die Karriereplanung etc. Vor allem aber finden sich in diesem Sektor zahlreiche neuartige, die kollektive Interessenvertretung ergänzende oder ersetzende Formen individueller Beteiligung in Form arbeitskräftebezogener Vereinbarungen zur Arbeitszeit (vgl. z.B. Böhm/Herrmann/Trinczek 2002; Sauer 2002), zu erfolgsbezogener Entlohnung (Heidemann 2000) usw.

Gerade auch in den Unternehmen der »volatilen « Dienstleistungsökonomie lassen sich innovative, durch Informalisierung und Individualisierung geprägte Formen der Interessenvertretung ausmachen, zumal diese Teilbranche - und dies gilt durchgängig für alle Unternehmensgrößen - als ausgesprochen mitbestimmungsarm ${ }^{7}$ einzustufen ist (Schmierl 2005). ${ }^{8}$ Dabei handelt es sich vorwiegend um nicht bis kaum institutionalisierte, eher dezentral von Belegschaftsgruppen oder Geschäftsleitung eingerichtete Ansätze der Vermittlung von Mitarbeiterinteressen, die nicht selten lediglich anlassbezogen eingerichtet werden (Städtler/ Feseker/Lange 2004). Dazu werden sog. »Mentoren« oder »Coaches« eingesetzt, neuartige Verhandlungsplattformen in Form von »Round Tables« oder von Projektnetzwerken installiert (Dowe u.a. 2001; Windeler/Wirth/Sydow 2001) oder Arbeitnehmerrepräsentanten im Aufsichtsrat mit der Ausübung betriebsratsähnlicher Nebentätigkeiten befasst (Classen/ Koller 2001). Die klassische Gegenüberstellung von Kollektivität versus Individualität als Kategorie der Beschreibung von Mustern der Arbeitsregulierung versagt daher bei der Analyse und Bewertung solch neuer Formen von Interessenartikulation und -durchsetzung.

Als exemplarisch für derartige Formen innovativer Interessenvertretung erweist sich eine Fallstudie in einem internationalen Softwarekonzern mit deutschem Stammsitz. ${ }^{9}$ Das dort entwickelte und seit mehreren Jahren praktizierte innovative Muster industrieller Beziehungen zeichnet sich besonders durch Momente von Entgrenzung, Individualität und Informalität aus und korrespondiert so mit der Betriebs- und Personalstruktur des Unternehmens. So

7) In einer empirischen Untersuchung zur Internetwirtschaft fanden Städtler u.a. in $11 \%$ der befragten Unternehmen Betriebsräte vor, in $18 \%$ waren alternative Gremien gegründet worden und $71 \%$ der befragten Beschäftigten arbeiteten in einem Unternehmen ohne Mitbestimmungsgremium. Auch verbreitete Annahmen, Krisenerfahrungen würden diese Beschäftigten den Gewerkschaften in die Arme treiben, konnten nicht bestätigt werden (2004).

8) Diese Entwicklungen verweisen offensichtlich auch darauf, dass der in der Theorie Reflexiver Modernisierung diagnostizierte Trend einer zunehmenden Individualisierung auch am System industrieller Beziehungen nicht spurlos vorübergegangen ist (Beck 2005; Kratzer 2005). 
sind zum einen in verschiedenen, durchaus im Geltungsbereich des deutschen Mitbestimmungsrechtes gelegenen Unternehmensteilen völlig unterschiedlich verfasste - z.T. komplementäre - Formen der betrieblichen Interessenvertretung etabliert; diese reichen von der dominanten Form individueller Interessendurchsetzung über verschiedene Formen informeller Mitarbeitervertretung bis zur klassischen Interessenvertretung qua Betriebsrat. Zum anderen entsprechen die Formen der informellen Interessenvertretung, die insbesondere bei der Mitbestimmung im Gesamtunternehmen gehandhabt werden, einer Belegschaft, die sich zu $85 \%$ aus Akademikern vor allem technischer Provenienz zusammensetzt und ein Durchschnittsalter von 34 Jahren aufweist - einer Arbeitnehmergruppe also, deren Bedürfnisse und Intentionen vorrangig auf die individuelle Durchsetzung eigener Interessen (hinsichtlich Karriere, Selbstorganisation etc.) gerichtet sind. Dies wiederum deckt sich weitgehend mit dem Interesse der Arbeitgeberseite an Mitarbeitern, die sowohl hochqualifiziert sind als auch über »soft skills« wie Artikulationsfähigkeit, Kommunikationskompetenzen und Verhandlungsgeschick verfügen und so auch im Rahmen einer offenen und direkten, ggf. Hierarchiestufen überspringenden Kommunikation ihre Interessen weitgehend selbst vertreten können.

Die dort praktizierten Formen kollektiver Interessenvertretung bleiben im Prinzip ohne instititutionell-rechtlichen Bezug auf das Betriebsverfassungsgesetz. Vielmehr konstituiert sich das informelle Vertretungsgremium des Kernunternehmens aus den Arbeitnehmervertretern im Aufsichtsrat. Angesichts des inzwischen erfolgten starken Unternehmens- und Belegschaftswachstums bot sich so für die Geschäftsleitung die Möglichkeit, diese zu einer betrieblichen Mitarbeitervertretung »umzufunktionieren«, die trotz fehlender betriebsverfassungsrechtlicher Kompetenzen in der Lage sein sollte, den im Großunternehmen unvermeidlichen Bedarf an einer Belegschaftsinteressenvertretung informell zu kanalisieren. Zugleich konnte mit diesem - eher konsens- denn konfliktorientierten - Modell dem immer wieder aufkommenden Ruf nach einem Betriebsrat begegnet werden. Aufgrund dieser Vertretungsstruktur und angesichts der seit längerem anhaltenden Prosperitätsphase beschränken sich die Aufgabenbereiche der informellen Mitarbeitervertretungen auf wenige Themen, während zahlreiche betriebsratstypische Verhandlungsfelder wie etwa Gesundheits- und Arbeitsschutz, Weiterbildung, Umweltschutz wegfallen. Letzteres gilt vor allem auch für das Verhandlungsfeld Gehalt und Entlohnungssystem, das - abgesehen von Rahmenregelungen der individuellen Verhandlung zwischen Vorgesetztem und einzelnem Mitarbeiter vorbehalten bleibt. Die Mitarbeitervertreter befassen sich vielmehr mit Problemfeldern, die sich aus dem und im individuellen Arbeitsverhältnis ergeben: Aufhebung oder Kündigung von Arbeitsverträgen, interne Personalumsetzung und Projektbesetzung, Mobbing oder Konflikte um Zielvereinbarungen bzw. Zielerreichung; dabei gleicht die Funktion des Arbeitnehmervertreters weniger der eines klassischen Interessenvertreters als vielmehr der eines individuellen Beraters oder eines Beistands in Mitarbeitergesprächen, oft auch nur der eines Moderators zwischen Mitarbeiter und Vorgesetztem.

Gleichwohl kann hier von einer Emergenz innovativer Formen industrieller Beziehungen gesprochen werden. Denn solche Formen der informellen Mitarbeitervertretung, die z.T. bereits über ein Jahrzehnt Bestand haben, erfahren eine Verfestigung insbesondere dadurch, dass ihnen institutionelle, wenn auch rechtlich nicht abgesicherte Qualitäten verliehen werden, etwa durch mit der Geschäftsleitung getroffene schriftliche Vereinbarungen. Obwohl die damit eingeräumten Mitwirkungsmöglichkeiten nicht justitiabel sind, bedeuten solche betriebsspezifischen Selbstverpflichtungen der Unternehmensleitung eine institutionelle An-

9) Das hier beschriebene empirische Fallbeispiel kann durchaus protoypisch für weitere von uns untersuchte große Firmen insbesondere aus der IT-Branche stehen, in welchen auf eine Betriebsratsgründung zugunsten einer Behandlung von Belegschaftsinteressen im Aufsichtsrat verzichtet wird (vgl. Schmierl 2005). 
erkennung, mit der den Mitarbeitervertretern faktisch ein größeres Durchsetzungspotenzial vermittelt wird. Allerdings finden sich auch empirische Anhaltspunkte dafür, dass im Zuge jüngerer, mit Einstellungsstopp, Kosteneinsparungen und schärferer Leistungskontrolle einhergehender Konsolidierungsprozesse die Funktionalität und Stabilität dieser Interessenvertretungsformen in Zweifel gezogen werden. Einerseits wird kritischer hinterfragt, inwieweit die bestehende Mitarbeitervertretung nicht doch nur eine Schönwetter-Lösung sei, die im Falle kritischer oder gar existenzbedrohender Unternehmenslagen, insbesondere im Falle drohender Standortschließungen oder Kündigungswellen, ungeeignet sein könne, in ausreichender Weise den Belegschaftsinteressen zur Durchsetzung zu verhelfen. Andererseits ist $\mathrm{zu}$ beobachten, dass Spielräume für eine erfolgreiche individuelle Interessendurchsetzung enger werden, weil die direkte Kommunikation über (mehrere) Hierarchieebenen sukzessive an Bedeutung verliert. Zudem sind Bürokratisierungstendenzen unverkennbar: Entscheidungsprozesse verlängern sich oder es werden angesichts drohender Karrierestaus weitere Hierarchieebenen in die in derartigen Unternehmen eher flachen Hierarchiestrukturen eingezogen. All dies schränkt die offene Kommunikationskultur offensichtlich zusehends ein und könnte dazu beitragen, die Funktionsfähigkeit eines derart auf individuelle und informellkollektive Interessenvertretung gestützten Modells zu untergraben.

Über die Beständigkeit solch innovativer, herkömmliche Grenzziehungen überschreitender Muster und Formen von Interessenvertretung besteht gegenwärtig also noch erhebliche Unklarheit. Offen ist vor allem, welche Verbreitung sie entfalten, wie sie auf die bekannten Institutionen der Arbeitsregulierung zurückwirken und ob sich daraus neuartige und vom bislang Selbstverständlichen grundlegend abweichende Formen oder aber die duale Interessenvertretung wieder stabilisierende Elemente entwickeln (vgl. Martens 2004). Dafür wird entscheidend sein, welcher Grad an institutioneller Verfasstheit der dabei praktizierten Festlegungen letztlich erreicht wird und inwieweit sich die Akteure der bisherigen kollektiven Interessenvertretung für solche innovativen Elemente öffnen (können und wollen). Nicht zuletzt wird dabei auch ausschlaggebend sein, welche Impulse und Effekte sich aus den jüngeren arbeitsrechtlichen Neuerungen (Reform des Betriebsverfassungsgesetzes, neue Tarifverträge über Weiterbildung etc.) für die institutionelle Qualität neuartiger Formen kollektiver und individueller Interessenvertretung ergeben, mit welcher Konsequenz also welche neuen Regelungen des Gesetzes in der Praxis umgesetzt werden. ${ }^{10}$

\section{Ausblick: Trotz offener Grenzziehungen und schwächerer Institutionalisie- rung mehr Arbeitnehmerbeteiligung?}

Insgesamt geht aus den hier skizzierten Entwicklungen deutlich hervor, dass die Auflösung der Strukturen und Grenzziehungen, die das System der industriellen Beziehungen in Deutschland bislang geprägt haben, in den vergangenen Jahren erheblich fortgeschritten ist und der Einfluss und die Reichweite der traditionellen inner- und überbetrieblichen Interessenvertretungen spürbar abgenommen haben. Unverkennbar ist aber auch, dass innerhalb dieses Erosionsprozesses zugleich, wenn auch z.T. zeitlich versetzt, Tendenzen zu beobachten sind, die einer solchen Auflösung zuwiderlaufen oder zumindest modifizierende Wirkung entfalten.

10) Nach ersten Erfahrungen mit dem neuen BetrVG nach der BR-Wahl 2002 hat die Zahl der Betriebsratsgremien und Betriebsratsmitglieder gegenüber 1998 jeweils um ca. $11 \%$ zugenommen und die Zahl der freigestellten Betriebsratsmitglieder sogar um $35 \%$, der tendenzielle Verlust an Betriebsratsgremien konnte also ausgeglichen werden, auch wenn die Verbreitung von Betriebsräten mit 12 $\%$ aller betriebsratsfähigen Betriebe immer noch auf einem niedrigen Niveau verharrt (vgl. Wassermann 2002; Handelsblatt vom 19.9.2002, S. 4). 
$\mathrm{Zu}$ konstatieren sind auf dem Gebiet der industriellen Beziehungen tendenziell vielfältigere und offenere Grenzziehungen und eine schwächere institutionelle Festigung, nicht immer nur zum Nachteil der betroffenen Arbeitnehmer. Letzteres wird deutlich, wenn Grenzüberschreitungen durch die Interessenvertretungsakteure etwa eine Ausweitung der Verhandlungsfelder, die Ausdehnung des Vertretungsbereichs auf ein größeres Klientel oder die innerorganisatorische Erschließung fachlicher und überfachlicher Kompetenzen zur Nutzung echter Kooperations- und Synergiepotentiale erlauben.

Dabei ist freilich nicht gewährleistet, dass durch veränderte Grenzziehungen und durch den Umgang mit innovativen Formen der Interessenvertretung der entgrenzungsbedingte Verlust herkömmlicher Schutzwirkungen und Verhandlungsfelder auf Dauer kompensiert werden kann. Denn auch wenn die herkömmlichen Strukturen und Formen der Interessenvertretung bis auf weiteres noch Fundament, Richtschnur und Maßstab für das deutsche System industrieller Beziehungen darstellen, sind doch zweifellos auch erhebliche Einbußen an Handlungspotenzial der Interessenvertreter der Arbeitnehmer zu verzeichnen, ist ihr Einfluss in vielen Betrieben gesunken oder völlig weggefallen, befinden sich zahlreiche Betriebsräte und Gewerkschafter nur noch in einer passiven Verteidigungsposition.

Umgekehrt bilden gerade die herkömmlichen Strukturen die Folie, von der sich neue Formen der Interessenvertretung abheben und gegenüber der sie sich konturieren müssen, wollen sie deren Schwächen und Rigiditäten hinsichtlich der Bewältigung der Anforderungen einer neuen Arbeitswelt überwinden. Dies wird nicht zuletzt in Wirtschaftsbereichen eine Rolle spielen, in denen Interessenvertretung und Mitbestimmung traditionell stark unterbelichtet sind, nämlich im Dienstleistungs- und KMU-Bereich. Denn in diesen Bereichen, die insbesondere durch die Entwicklungen in der New Economy an wirtschaftlichem Gewicht deutlich zugenommen, inzwischen aber auch an Berichten über Arbeitsplatzkonflikte »aufgeholt« haben, sind verstärkt Neuerungen der Interessenvertretung zu erkennen, die von der erstmaligen Bildung von Betriebsräten alter Prägung (mit neuer Orientierung) bis zu völlig informellen und individualisierten Konstruktionen reichen. ${ }^{11}$

$\mathrm{Ob}$ sich hinter solchen Neujustierungen von Grenzziehungen dauerhafte Ansätze für eine anders gefügte Welt der Interessenvertretung verbergen, in denen sich gar reflexiv moderne Elemente industrieller Beziehungen ankündigen, oder ob es sich dabei um typische Offenheiten experimenteller Innovationen handelt, kann bislang noch nicht beantwortet werden. Auch wenn wir die mit dem Entgrenzungsprozess sich vollziehende Pluralisierung, Differenzierung und Individualisierung der Interessenvertretungsformen als Hybridisierung des deutschen Systems der industriellen Beziehungen $\mathrm{zu}$ fassen suchen (vgl. Schmierl 2001; Heidling u.a. 2004), ${ }^{12}$ bleibt die mittel- und langfristige Perspektive gleichwohl offen: Handelt es sich bei der gegenwärtigen Gleichzeitigkeit von Kontinuität und Diskontinuität alter und neuer Grenzziehungen um eine auf Dauer gestellte Hybridsituation, um den Übergang zu einer neuen, durch andersartige Basisinstitutionen und -prinzipien geprägten Konstellation oder nur um Auflösungserscheinungen im Zuge eines sich fortentwickelnden Modernisierungsprozesses?

Die Klärung dieser Fragen bedarf angesichts dessen, dass die innovativen Formen der Interessenvertretung noch wenig verbreitet und konsolidiert sowie in ihrer Effizienz noch wenig untersucht sind, vielfältiger Analysen auf theoretisch-konzeptioneller wie auf empirischer Ebene. Zuverlässige, gar prognostische Aussagekraft entfaltende Einschätzungen

11) Vgl. dazu Ergebnisse einer Befragung von Nemax-Unternehmen durch die Ruhr-Universität Bochum (Ittermann/Niewerth 2004), wonach in $40 \%$ der Unternehmen, die geantwortet haben, Betriebsräte eingerichtet waren - aber fast ebenso viele Fälle mit Selbstvertretungsmodellen angetroffen wurden, wo verschiedenste andere Formen etwa des Round Table, des Coachings etc. praktiziert werden. 
verbieten sich daher zum gegenwärtigen Zeitpunkt. Allerdings kann u.E. zumindest bereits jetzt gesagt werden, dass drei im Kontext der Entgrenzungsanalyse deutlich gewordene Zusammenhänge für die Beantwortung dieser grundsätzlichen Fragen von entscheidender Relevanz sein dürften.

(1) Einerseits muss im Rückblick auf unsere Ausgangsüberlegungen festgehalten werden, dass jegliche - sei es durch Auflösung, sei es durch Emergenz - veränderte Grenzbildung ambivalente Wirksamkeit entfaltet, mit der für die Akteure und Betroffenen der industriellen Beziehungen jeweils unterschiedliche Konsequenzen verbunden sein können. Weil Grenzziehungen das Handeln und den Einfluss von gesellschaftlichen Akteuren sowohl begrenzen als auch ermöglichen, können rigide Grenzlinien hohe Schutzwirkungen, aber auch stark eingeengte Handlungsspielräume bedeuten, während offenere und vielfältigere Grenzziehungen weniger Schutz bieten, jedoch auch größere Einflussnahme und leichtere Grenzüberschreitungen zulassen können. Zudem dienen strikte institutionelle Grenzziehungen auch der Reduktion von Komplexität, indem sie Klarheit hinsichtlich Teilhabeansprüchen und Einflussbereichen schaffen und die Rechtssicherheit für alle Beteiligten erhöhen. Das bedeutet umgekehrt, dass flexiblere Grenzziehungen eine höhere Komplexität in den Beziehungsgeflechten, in den Entscheidungs- und Mitwirkungsprozessen sowie eine größere Intransparenz in den Aushandlungs- und Regulierungsresultaten und deren Reichweite und Stabilität mit sich bringen.

Mit anderen Worten: Je größer die strukturbildende Kraft und die institutionelle Relevanz von Grenzziehungen ausfällt, umso größere Gewissheit und Verlässlichkeit erzeugen sie für die Kollektivakteure und die Beschäftigten, um so mehr nehmen Gewissheit und Planbarkeit - für beide Parteien der industriellen Beziehungen - zu, umso zwingender und eingeschränkter sind aber auch die Spielräume und Verhandlungsfelder, die ihnen zur Verfügung stehen; je vielfältiger, offener und weniger institutionalisiert solche Grenzlinien sind, umso höher sind die Risiken für die Beteiligten, umso größer sind aber auch ihre Chancen, jenseits dieser Grenzen gelegene Möglichkeiten zu nutzen.

(2) Inwieweit beides in der Realität zum Vorteil oder zum Nachteil der Interessenvertretungsakteure bzw. der von ihnen repräsentierten Arbeitnehmer gereicht, hängt nicht nur vom Grad der Auflösung alter bzw. der Herausbildung neuer Grenzziehungen und deren jeweiliger institutioneller Ausprägung ab. Über die gesellschaftlich strukturierende Kraft und den Einfluss des Systems industrieller Beziehungen entscheiden auch die Verhandlungspotenziale bzw. Macht- und Rechtspositionen, derer sich die Akteure der Interessenvertretungen im Rahmen der Interessenvertretungsformen bedienen können. Verhandlungsstärke und Konfliktbereitschaft (innerhalb der jeweiligen betrieblichen Rahmenbedingungen) gründen auf

12) In der Terminologie der Theorie Reflexiver Modernisierung könnte man diese Hybridisierung im Feld industrieller Beziehungen im Hinblick auf die Erscheinungsformen des Neuen auch als »hierarchisch geordneten Pluralismus« (Beck/Bonß/Lau 2004, S. 35ff.) bezeichnen: Das tradierte System industrieller Beziehungen mit seinen Besonderheiten: duale Interessenvertretungsstruktur, starke Zentralität, kollektive Interessenvertretungsrepräsentanz sowie einem hohen Grad an Verrechtlichung (Schmierl 2001) sieht sich zunehmend neben andere, konkurrierende Modi von Interessenvertretung gestellt. Die abweichenden Optionen lassen sich nicht mehr nur als künftig absorbierbare Randphänomene marginalisieren, sondern müssen als keinesfalls mehr zu vernachlässigende neue Erscheinungsformen betrachtet werden. Allerdings bleibt - zumindest bis auf weiteres - das klassische, hochgradig mitbestimmte Modell diesen Abweichungen gegenüber als übergeordnet, »normal « und vorgezogen anerkannt. Da diesem Typus und seinen internen Differenzierungen interessen- und berufspolitische sowie ökonomische Konkurrenzbeziehungen immanent sind, ist mit Instabilitäten zu rechnen, sodass gegenwärtig auch eine Aussage zu den Zukunftsperspektiven weit mehr als wissenschaftlich-prognostische Fähigkeiten voraussetzen würde. 
Solidarisierungspotenzialen in und zwischen den Betrieben, auf Kompetenzen und Informationswissen sowie auf der Verfügbarkeit geeigneter Mobilisierungsinstrumente.

Inhaltliche, organisatorische, klientelbezogene und institutionelle Grenzziehungen konditionieren die Einflusspotentiale der Interessenvertretungen also sicherlich in gewisser Weise. Ob sich damit allerdings eine Schwächung oder eine Chancenvergrößerung für die traditionellen Kollektivakteure verbindet bzw. ob Arbeitnehmervertreter innerhalb neuer informeller und individualisierter Formen der Interessenvertretung erfolgreich agieren, hängt aber auch davon ab, inwieweit für die Nutzung dieser Offenheiten im Interesse der Arbeitnehmer geeignete Handlungs- bzw. Mitwirkungspotenziale zur Verfügung stehen, und zwar auf Grundlage der betrieblichen Rahmenbedingungen, der Kompetenzen, der Verfügbarkeit über Wissen, innerbetrieblich mobilisierbarer Solidarität, der Möglichkeit zu überbetrieblicher Kooperation etc. Je offener und flexibler also Grenzziehungen gestaltet sind, umso bedeutsamer werden Macht- und Handlungspotenziale auf der Grundlage von Solidarität und Kompetenzen. Partizipative Arbeitnehmerbeteiligung wird im Rahmen individualisierter und informeller Formen vor allem dort thematisiert, wo das Vertretungsprinzip in Richtung auf Interessenmediation und Koordination durch die Kollektivakteure aufgelockert wird und individualisierte Elemente der Interessendurchsetzung ein größeres Gewicht erhalten. Gleichwohl wird auch hierfür ein gewisser Grad an geordneten Strukturen und verlässlichen Verfahren, sprich an Grenzziehungen unabdingbar sein, damit individualisierten und informellen Formen der Interessenvertretung und den darauf basierenden Verhandlungsergebnissen die notwendige konjunkturfeste Stabilität und Anerkennung bei den Arbeitnehmern verliehen wird.

(3) Das jeweilige Gefüge von Grenzziehungen im System der industriellen Beziehungen stellt also gewissermaßen das Gerippe, das Skelett für traditionelle ebenso wie für neue informelle Akteure der Interessenvertretung dar, um das herum Handlungspotenziale zu entfalten sind; dieses Gefüge kann sich aber auch als Korsett erweisen, wenn dadurch Handlungsoptionen erschwert oder verbaut werden. Mit diesem Spannungsverhältnis bzw. mit der damit verbundenen Notwendigkeit zur Herstellung einer Balance aus beiden Momenten müssen sich letztlich auch alle innovativen oder in Anpassung befindlichen Formen der Interessenvertretung auseinandersetzen, wenn damit bei verflüssigten Grenzziehungen eine erfolgreiche Vertretung und Durchsetzung von Arbeitnehmerinteressen erreicht werden soll. Das heißt also, dass neue Formen der Interessenvertretung auch dann ein Gleichgewicht zwischen beiden Aspekten finden müssen, wenn sie zwar stark informell und individualisiert geprägt sind und damit Flexibilitätsvorteile bieten, gleichwohl aber im Interesse der Vertretenen ein Minimum an institutioneller Grenzziehung zum Schutz etwa von Verhandlungsprozeduren und der dauerhaften Gültigkeit von Vereinbarungen gewährleisten sollen. Dabei kann es angesichts der Vielfalt neuer Interessenvertretungsmuster zu durchaus unterschiedlichen Balancen zwischen Schutzorientierung und Offenheitsopportunitäten kommen.

Eine derartige Ausgewogenheit müssen innovative Formen der Interessenvertretung erst recht aufweisen, wenn sie als neue Grundmuster der industriellen Beziehungen strukturprägende Bedeutung erfahren sollen. Grenzziehungen und mobilisierbare Handlungspotenziale müssen dann in einem Maße erprobt und austariert sein, dass einerseits der nötige Schutz für die Arbeitnehmer durchsetzbar wird, andererseits aber die für Verhandlungen und Regularien angestrebten Flexibilitätsspielräume auch nachhaltig verfügbar bleiben, ohne dass die Konstellation bei veränderten ökonomischen oder organisationalen Rahmenbedingungen gleich aus der Balance gerät.

Vor allem wenn wir klären wollen, wie beständig innovative, herkömmliche Grenzziehungen der Interessenvertretung überschreitende Muster der Interessenvertretung sind, inwieweit sich daraus neuartige und vom bislang Selbstverständlichen abweichende oder die duale 
Interessenvertretung wieder stabilisierende Elemente entwickeln und welcher Grad an institutioneller Verfasstheit dafür notwendig ist, muss in den weiteren Analysen zum Feld industrieller Beziehungen gerade diesen für eine Balance entscheidenden Momenten in der Grenzgestaltung und in den Handlungspotentialen nachgegangen werden. Wesentlich wird vor allem sein, inwiefern bei weichen Grenzziehungen das Schutz- und Vertretungsinteresse der Arbeitnehmerschaft ausreichend erfüllt werden kann, ohne dass Pluralität und Offenheit dadurch wieder eingeschränkt werden. Die Momente dieser notwendigen Balance zu identifizieren und in ihren Effekten einzuschätzen, insbesondere zu bestimmen, ob und welche Elemente industrieller Beziehungen den Interessenvertretungsformen zu Stabilität und nachhaltiger Wirksamkeit trotz oder gerade wegen ihrer Offenheit, Vielfalt und Flexibilität verhelfen, wird eine der Hauptaufgaben unserer zukünftigen Forschungsarbeiten sein.

\section{Literatur}

Abel, J./Sperling, H.J. (Hrsg.) (2000): Umbrüche und Kontinuitäten, München/Mering.

Abel, J./Ittermann, P./Wannöffel, M. (2001): Alte und neue Arenen der industriellen Beziehungen - Resümee und Ausblick. In: J. Abel/H.J. Sperling (Hrsg.): Umbrüche und Kontinuitäten, München/ Mering, S. 383-405.

Altmann, N./Deiß, M. (1996): Arbeit in der vernetzten Produktion. In: D. Schulte (Hrsg.): Arbeit der Zukunft. Beiträge zur Reformdiskussion im Deutschen Gewerkschaftsbund und seinen Gewerkschaften, Band 5, Köln, S. 84-103.

Artus, I. (2004): Testfeld Ost? Betriebliche Interessenvertretung in Ostdeutschland - Konsequenzen für den Westen. In: WSI-Mitteilungen 57, S. 271-276.

Beck, U. (2005): Gibt es eine europäische Gesellschaft? Kritik des methodologischen Nationalismus oder Perspektiven einer kosmopolitischen Soziologie. In: K.-S. Rehberg (Hrsg.): Soziale Ungleichheit Kulturelle Unterschiede. Verhandlungen des 32. Kongresses der Deutschen Gesellschaft für Soziologie in München. Frankfurt/New York (im Erscheinen).

Beck, U./Bonß, W./Lau, Ch. (2004): Entgrenzung erzwingt Entscheidung: Was ist neu an der Theorie reflexiver Modernisierung? In: U. Beck/Ch. Lau (Hrsg.): Entgrenzung und Entscheidung, Frankfurt, S. 13-62.

Beese, B./Dörre, K./Röttger, B. (2004): Von Seilschaften zu innovativen Netzwerken? Strukturwandel, regionale Industriepolitik und die Gewerkschaften. In: FIAB (Hrsg.): Jahrbuch Arbeit, Bildung, Kultur, Bd. 21/22, Recklinghausen, S. 9-37.

Bender, G. (1997): Lohnarbeit zwischen Autonomie und Zwang - Neue Entlohnungsformen als Element veränderter Leistungspolitik. Frankfurt/New York.

Benz-Overhage, K. (2000): Neue Anforderungen an die Betriebspolitik. In: WSI-Mitteilungen 53, S. 4655 .

Bispinck, R. (2004): Kontrollierte Dezentralisierung der Tarifpolitik - Eine schwierige Balance. In: WSIMitteilungen 57, S. 237-245.

Böhle, F./Bolte, A./Drexel, I./Dunkel, W./Pfeiffer, S./Porschen, S. (2002): Umbrüche im gesellschaftlichen Umgang mit Erfahrungswissen - Theoretische Konzepte, empirische Befunde, Perspektiven der Forschung. München: ISF München Forschungsberichte.

Böhm, S./Herrmann, C./Trinczek, R. (2002): Löst Vertrauensarbeitszeit das Problem der Vereinbarkeit von Familie und Beruf? In: WSI- Mitteilungen 55, S. 435-441.

Boes, A./Döhl, V./Kratzer, N./Marrs, K./Sauer, D. (2001): Die Auflösung des Unternehmens. Zur Entgrenzung von Kapital und Arbeit - Grenzen der Entgrenzung. Teilprojekt C1 des Sonderforschungsbereichs $536 »$ Reflexive Modernisierung - Analysen zur Transformation der industriellen Moderne«, München, S. 613-663.

Bosch, G./Hennicke, P./Hilbert, J./Kristof, K./Scherhorn, G. (Hrsg.) (2002): Die Zukunft von Dienstleistungen, Frankfurt/New York. 
Brandl, S./Lawatsch, U. (1999): Vernetzung von betrieblichen Interessenvertretungen entlang der Stoffströme. Discussion Paper des WZB, Berlin.

Classen, H./Koller, B. (2001): Wir sind Quasibetriebsräte. Interview in: Mitbestimmung 47, S. 48-51.

Deiß, M. (2000): Betriebsrat - Quo vadis? Interessenvertretung in vernetzten Wertschöpfungsketten. In: H. Betz/M. Möreke/U. Klitzke (Hrsg.): Vom Klassenkampf zum Co-Management, Hamburg, S. 117-146.

Deiß, M./Heidling, E. (2001): Interessenvertretung und Expertenwissen - Anforderungen und Konsequenzen für Betriebsräte und Gewerkschaften, Düsseldorf.

Deiß, M./Mendius, H.G. (2005): EU-Osterweiterung und Arbeitnehmer - Das Beispiel der bayerischtschechischen Grenzregion, Marburg.

Döhl, V./Kratzer, N./Moldaschl, M./Sauer, D. (2001): Die Auflösung des Unternehmens? Zur Entgrenzung von Kapital und Arbeit. In: U. Beck/W. Bonß (Hrsg.): Die Modernisierung der Moderne, Frankfurt, S. 219-232.

Dowe, C./Welzel, C./Stradtmann, P./Bieber, C./Scharfenberg, H. (2001): »Are we family?!« Umfang und Formen der Mitarbeiter-Mitbestimmung in der New Economy, Berlin.

Duden (2000): Die deutsche Rechtsschreibung, Band 1, Mannheim.

Eichmann, H. (2004): Arbeitskraftunternehmer in der New Economy. In: H.J. Pongratz/G.G. Voß: Typisch Arbeitskraftunternehmer? Berlin, S. 73-92.

Ellguth, P. (2004): Erosion auf allen Ebenen? Zur Entwicklung der quantitativen Basis des dualen Systems der Interessenvertretung. In: I. Artus/R. Trinczek (Hrsg.): Über Arbeit, Interessen und andere Dinge, München/Mering, S. 159-179.

Ellguth, P./Kohaut, S. (2004): Tarifbindung und betriebliche Interessenvertretung: Ergebnisse des IABBetriebspanels 2003. In: WSI-Mitteilungen 57, S. 450-454.

Funder, M. (2000): Entgrenzung von Organisationen - eine Fiktion? In: H. Minssen (Hrsg.): Begrenzte Entgrenzungen, Berlin, S. 19-45.

Gerst, D. (2004): Industrielle Gruppenarbeit und der Leittypus des Arbeitskraftunternehmers. In: H.J. Pongratz/G.G. Voß: Typisch Arbeitskraftunternehmer? Berlin, S. 187-207.

Gesterkamp, T. (2000): Vom Betriebsrat zum Netzrat. In: Mitbestimmung 46, S. 59.

Hartmann, M. (2002): Widersprüche, Ambivalenzen, Paradoxien - Begriffliche Wandlungen in der neueren Gesellschaftstheorie. In: A. Honneth (Hrsg.): Befreiung aus der Mündigkeit, Frankfurt/New York, S. 221-251.

Hassel, A. (2000): Organisationsreform und Organisationsformen. In: Gewerkschaftliche Monatshefte 51, S. 129-139.

Heidemann, W. (2000): Weiterentwicklung und Mitbestimmung im Spiegel betrieblicher Vereinbarungen. Auswertung von Betriebs- und Dienstvereinbarungen, Düsseldorf.

Heidling, E./Deiß, M./Meil, P./Schmierl, K. (2004): Restrukturierung nationaler Interessenvertretung. In: U. Beck/W. Bonß/Ch. Lau (Hrsg.): Entgrenzung und Entscheidung: Perspektiven reflexiver Modernisierung, Frankfurt, S. 360-383.

Hielscher, V. (2000): Entgrenzung von Arbeit und Leben? Die Flexibilisierung von Arbeitszeiten und ihre Folgewirkungen für die Beschäftigten. Discussion Paper des WZB FS II 00-201, Berlin.

Hlawna, R. (2001): Der große Mythos. In: Mensch und Büro, Heft 3, S. 38-42.

Höpner, M. (2003): Wer beherrscht die Unternehmen? Frankfurt/New York.

Ittermann, P./Niewerth, C. (2004): »Bleibt alles anders?« Organisations- und Beteiligungsstrukturen in der digitalen Wirtschaft - Ergebnisse einer repräsentativen Befragung, SOAPS-Papers Nr. 4, 3. Jg.

Jurczyk, K./Voß, G. (2000): Entgrenzte Arbeitszeit - Reflexive Alltagszeit. Die Zeiten des Arbeitskraftunternehmers. In: E. Hildebrandt (in Zusammenarbeit mit G. Linne) (Hrsg.): Reflexive Lebensführung, Berlin, S. 151-206.

Keller, B. (2004): Zusammenschlüsse von Gewerkschaften. Folgen und Perspektiven am Beispiel der Vereinten Dienstleistungsgewerkschaft (ver.di). In: Leviathan 32, S. 89-112. 
Kohaut, S./Schnabel, C. (2003): Zur Erosion des Flächentarifvertrags: Ausmaß, Einflussfaktoren und Gegenmaßnahmen. In: Industrielle Beziehungen 10, S. 193-219.

Kratzer, N. (2001): Entgrenzung von Arbeit - Neue Leitbilder der Erwerbsarbeit? Ansätze zur arbeitspolitischen Bewertung und Gestaltung, hektogr. Endbericht, München.

Kratzer, N. (2003): Arbeitskraft in Entgrenzung - Grenzenlose Anforderungen, erweiterte Spielräume, begrenzte Ressourcen, Berlin.

Kratzer, N. (2005): Vermarktlichung und Individualisierung. Zur Produktion sozialer Ungleichheit in der Zweiten Moderne. In: Soziale Welt 56, S. 245-264.

Kühl, St. (1998): Wenn die Affen den Zoo regieren - Die Tücken der flachen Hierarchien, Frankfurt/New York.

Lau, C./Gugutzer, R./Viehöver, W./Keller, R. (2002): Vergesellschaftung der Natur - Naturalisierung der Gesellschaft. Teilprojekt A2 des Sonderforschungsbereichs $536 »$ Reflexive Modernisierung - Analysen zur Transformation der industriellen Moderne «. Arbeits- und Ergebnisbericht, München, S. 53-95.

Lompe, K./Blöcker, A./Marquart, B./Rölke, P./Weis, H. (2003): Bilanz und Perspektiven der Montanmitbestimmung, Berlin.

Martens, H. (2000): Die Netzwerkgewerkschaft - Eine Zukunftsoption angesichts von Krise und Verfall der alten Institutionen der Arbeit. In: G. Naegele/G. Peter (Hrsg.): Arbeit - Alter - Region, Münster, S. 271-287.

Martens, H. (2001): Herausforderungen an eine neue gewerkschaftliche Arbeitspolitik angesichts von Krise und Verfall der alten Institutionen der Arbeit. In: H. Martens/G. Peter/F. Wolff (Hrsg.): Zwischen Selbstbestimmung und Selbstausbeutung - gesellschaftlicher Umbruch und neue Arbeit, Frankfurt/ New York, S. 170-191.

Martens, H. (2004): Primäre Arbeitspolitik und gewerkschaftliche Interessenvertretung in der New Economy - empirische Befunde aus gewerkschaftlichen Modellprojekten. In: Arbeit 13, S. 166-172.

Meil, P./Heidling, E./Schmierl, K. (2003): Die (un-)sichtbare Hand. Nationale Systeme der Arbeitsregulierung in der Ära des Shareholder Value - Ein internationaler Vergleich: Deutschland, Frankreich, USA, Forschungsberichte aus dem ISF München.

Mensch und Büro (2001): Büroberater blicken in die Office-Zukunft. In: Heft 3, S. 126-127.

Minssen, H. (Hrsg.) (2000): Begrenzte Entgrenzungen - Wandlungen von Organisation und Arbeit, Berlin.

Moldaschl, M./Sauer, D. (2000): Internalisierung des Marktes - Zur neuen Dialektik von Kooperation und Herrschaft. In: H. Minssen (Hrsg.): Begrenzte Entgrenzungen - Wandlungen von Organisation und Arbeit, Berlin, S. 205-224.

Müller, H.-P. (2001): Die Mühen der Profilfindung einer Dienstleistungsgewerkschaft. Zur Entstehungsgeschichte der Multibranchengewerkschaft »Ver.di« im Spannungsfeld von Organisationskonflikten und Programmsuche. In: Industrielle Beziehungen 8, S. 108-137.

Müller-Jentsch, W. (2000): Wandel der Unternehmens- und Arbeitsorganisation und seine Auswirkungen auf die Interessenbeziehungen zwischen Arbeitgebern und Arbeitnehmern. In: M. Funder/H.P. Euler/ G. Reber (Hrsg.): Entwicklungstrends der Unternehmensreorganisation, Linz, S. 163-177.

Plehwe, D. (2001): Arbeitspolitische Probleme ungleicher Reorganisation. Zur Veränderung der Arbeit in Logistiknetzwerken. In: Industrielle Beziehungen 8, S. 55-82.

Pongratz, H.J./Voß, G.G. (2003): Arbeitskraftunternehmer - Erwerbsorientierungen in entgrenzten Arbeitsformen, Berlin.

Pongratz, H.J./Voß, G.G. (2004): Typisch Arbeitskraftunternehmer? Berlin.

Rehder, B. (2002): Wettbewerbskoalition oder Beschäftigungsinitiativen? In: H. Seifert (Hrsg.): Betriebliche Bündnisse für Arbeit, Berlin, S. 87-102.

Rehder, B. (2003): Betriebliche Bündnisse für Arbeit in Deutschland. Mitbestimmung und Flächentarif im Wandel. Schriftenreihe des MPI für Gesellschaftsforschung, Band 48, Frankfurt/New York.

Satzer, R. (2001): Nicht nur Traumjobs - Vom Arbeiten und Verdienen in den Medien. Frankfurt: connexx.av. 
Sauer, D. (2002): »Vertrauensarbeitszeit« und der Umbruch in der gesellschaftlichen Organisation von Arbeit. In: ver.di (Hrsg.): Vertrauensarbeitszeit - tarifpolitischer Workshop Berlin, S. 9-26.

Schmierl, K. (1995): Umbrüche in der Lohn- und Tarifpolitik. Neue Entgeltsysteme bei arbeitskraftzentrierter Rationalisierung in der Metallindustrie, Frankfurt/New York.

Schmierl, K. (1998): Amorphie im »Normierten Verhandlungssystem « - Wandel industrieller Beziehungen im internationalen Unternehmensverbund. In: M. von Behr/H. Hirsch-Kreinsen (Hrsg.): Globale Produktion und Industriearbeit, Frankfurt/New York, S. 163-207.

Schmierl, K. (2000): Interessenvertretung ohne kollektive Akteure? Grenzen industrieller Beziehungen bei entgrenzter Produktion. In: M. Funder/H.P. Euler/G. Reber (Hrsg.): Entwicklungstrends der Unternehmensreorganisation. Internationalisierung, Dezentralisierung, Flexibilisierung, Linz, S. 73-107.

Schmierl, K. (2001): Hybridisierung der industriellen Beziehungen in der Bundesrepublik - Übergangsphänomen oder neuer Regulationsmodus? In: Soziale Welt 52, S. 427-448.

Schmierl, K. (2003a): Vielfalt im Umbruch - Auflösungserscheinungen, Anpassungsprozesse und neue Interessenvertretungsmodelle in den Arbeitsbeziehungen. In: J. Beyer (Hrsg.): Vom Zukunftsmodell zum Auslaufmodell? Die deutsche Wirtschaftsordnung im Wandel, Wiesbaden, S. 36-60.

Schmierl, K. (2003b): Mitbestimmungsdilemmata in internationalisierenden kleinen und mittleren Unternehmen. In: M. von Behr/K. Semlinger (Hrsg.): Internationalisierung kleiner und mittlerer Unternehmen. Neue Entwicklungen bei Arbeitsorganisation und Wissensmanagement, Frankfurt/New York, S. 273-307.

Schmierl, K. (2004): Pluralisierung statt Auflösung - Industrielle Beziehungen im Anpassungsprozess. In: J. Allmendinger (Hrsg.) (2004): Entstaatlichung und soziale Sicherheit. Verhandlungen des 31. Kongresses der Deutschen Gesellschaft für Soziologie in Leipzig 2002. 2 Bände + CD-ROM.

Schmierl, K. (2005): Neue Muster der Interessendurchsetzung in der Wissens- und Dienstleistungsökonomie - Elemente einer Hybridisierung industrieller Beziehungen. In: R. Trinczek (Hrsg.): Arbeitsbeziehungen in Betrieben ohne Betriebsrat (Veröffentlichung in Vorbereitung).

Schmierl, K./Heidling, E./Meil, P./Deiß, M. (2001): Umbruch des Systems industrieller Beziehungen. In: U. Beck/W. Bonß (Hrsg.): Die Modernisierung der Moderne - Überlegungen zur Transformation der industriellen Moderne, Frankfurt, S. 233-246.

Schmierl, K./Pfeiffer, S. (2004): Die Lego-Logik der kapitalistischen »Netzwerkökonomie« - Theoretische Spekulationen zum Wandel von Betrieb und Technik. In: M. Faust/M. Funder/M. Moldaschl (Hrsg.): Die Organisation der Arbeit, München/Mering.

Schulze-Buschoff, K. (2000): Vom Normalarbeitsverhältnis zur Flexibilisierung - Über den Wandel der Arbeitszeitmuster: Ausmaß, Bewertung und Präferenzen. Discussion Paper des WZB P00-518, Berlin.

Seifert, H. (Hrsg.) (2002): Betriebliche Bündnisse für Arbeit, Berlin.

Sperber, K. (2002): Die Qual der Katholiken beim Urnengang. In: FR vom 5.9.2002, S. 5.

Städtler, A./Feseker, K./Lange, H. (2004): Arbeits- und Interessenregulierung in klein- und mittelständischen Unternehmen der digitalen Wirtschaft. In: Arbeit 13, S. 148-163.

Steinrücke, M./Spitzley, H./Raasch, S./Mückenberger, U./Hildebrandt, E. (Hrsg.) (2001): Neue Zeiten neue Gewerkschaften, Berlin.

Streeck, W./Höpner, M. (Hrsg.) (2003): Alle Macht dem Markt? Fallstudien zur Abwicklung der Deutschland AG, Frankfurt/New York.

Stumpf-Fekete, M. (2000): Regime Shopping und Arbeitnehmermitwirkung - Standortentscheidungen deutscher Konzerne in einer entgrenzten Wirtschaft. In: H. Minssen (Hrsg.): Begrenzte Entgrenzungen, Berlin, S. 71-82.

Sydow, J. (1999): Mitbestimmung in Unternehmungsnetzwerken. In: B. Frick/N. Kluge/W. Streeck (Hrsg.): Die wirtschaftlichen Folgen der Mitbestimmung, Frankfurt/New York, S. 171-222.

Sydow, J./Wirth, C. (1999): Arbeit, Personal und Mitbestimmung in Unternehmungsnetzwerken, München/Mering.

Trautwein-Kalms, G. (2001): Dienstleistungsarbeit - ein weites Feld für die Interessenvertretung. WSI Mitteilungen 54, S. 365-372. 
Viering, J. (2004): Mehr Vielfalt bei Mitbestimmung. In: Süddeutsche Zeitung vom 26. Oktober 2004, S. 24.

Voß, G. (1998): Die Entgrenzung von Arbeit- und Arbeitskraft - Eine subjektorientierte Interpretation des Wandels der Arbeit. In: MittAB 31, S. 473-487.

Wagner, A. (2001): Entgrenzung der Arbeit und der Arbeitszeit? In: Arbeit 10, S. 365-378.

Wassermann, W. (2002): Betriebsräte - Akteure für Demokratie in der Arbeitswelt, Münster.

Wehling, M. (2000): Mitbestimmung in virtuellen Unternehmungen. In: Industrielle Beziehungen 7, S. 131-156.

Wendeling-Schröder, U. (2001): Arbeitsrechtliche Aspekte der Novellierung des Betriebsverfassungsgesetzes. In: Gewerkschaftliche Monatshefte 52, S. 221-228.

Windeler, A./Wirth, C./Sydow, J. (2001): Die Zukunft in der Gegenwart erfahren. Arbeit in Projektnetzwerken der Fernsehproduktion. In: Arbeitsrecht im Betrieb 22, S. 12-18.

Wolf, H./Mayer-Ahuja, N. (2002): »Grenzen der Entgrenzung von Arbeit«-Perspektiven der Arbeitsforschung. In: SOFI-Mitteilungen 30, Göttingen, S. 197-205.

Dr. Manfred Deiß,Manfred.Deiss@isf-muenchen.de. Dr. Klaus Schmierl, Klaus.Schmierl@isf-muenchen.de. Institut für Sozialwissenschaftliche Forschung e.V. (ISF) München, Jakob-Klar-Str. 9, 80796 München. 